\title{
The Range of Validity for the Kelvin Force
}

(PRL 84, 2762, (2000))

In a recent Letter [1], Luo, Du and Huang reported a novel convective instability driven by a force rarely studied before - that exerted by an external magnetic field on a strongly magnetizable liquid. The associated physics is surprisingly rich and promises many more interesting results for the future. Unfortunately, the analysis starts from a misconception and employs the Kelvin force outside its range of validity. Since few would recognize this as a mistake, and since its consequence in the given experiment is particularly direct and critical, this is a point well worth being clarified, and clearly understood.

In the experiment, ferrofluid is exposed to a constant B-field. Yet, since the temperature $T$ and the density $\rho$ of magnetic particles vary, so does the magnetic field $\mathbf{H}=\mathbf{B} /[1+\chi(T, \rho)]$, giving rise to a finite Kelvin force. With $\chi$ the magnetic susceptibility and $\mathbf{M}=\chi \mathbf{H}$ the magnetization, this force is given as

$$
\mathbf{f}=M_{i} \boldsymbol{\nabla} H_{i}=\left(M_{i} B_{i}\right) \boldsymbol{\nabla} \frac{1}{1+\chi}=-\frac{(\chi B)^{2}}{(1+\chi)^{3}} \frac{\boldsymbol{\nabla} \chi}{\chi} .
$$

(Summation over the index $i$ is implied.) Eq (1) may be derived from the more general Helmholtz force [2],

$$
\mathbf{f}=+\boldsymbol{\nabla}\left(\frac{1}{2} H^{2} \rho \partial \chi / \partial \rho\right)-\frac{1}{2} H^{2} \nabla \chi,
$$

by considering a dilute ferrofluid, and taking $\chi$ as proportional to the particle density $\rho$, or $\rho \partial \chi / \partial \rho=\chi$. Then Eq (2) clearly reduces to $\mathbf{f}=\frac{1}{2} \chi \nabla\left(H^{2}\right)=M_{i} \nabla H_{i}$.

All this seems rather convincing, but in fact hides a pitfall. Closer scrutiny reveals that $\mathbf{f}=M_{i} \boldsymbol{\nabla} H_{i}$ is only valid to linear order in $\chi$. (Except in unconventional systems of more recent dates, the magnetic susceptibility $\chi$ is usually much smaller than 1 , so terms of higher order in $\chi$ have always been negligible. This may well be the reason why the confined range of validity of the Kelvin force has been such a well kept secret.) If true, the expressions of Eq (1) merely states that the force vanishes - to linear order in $\chi$. No result derived from Eq (1) is then trustworthy.

To qualitatively understand this restriction, define a different susceptibility, $\mathbf{M}=\tilde{\chi} \mathbf{B}$. With the permeability given as $\mu=1+\chi=(1-\tilde{\chi})^{-1}$, we have $\tilde{\chi}=\chi /(1+\chi)$. Both susceptibilities are clearly physically equivalent, and we have no a priori reason to prefer either. Employing $\mathrm{d} \tilde{\chi}=\mathrm{d} \chi /(1+\chi)^{2}$, we may rewrite Eq (2) as

$$
\mathbf{f}=+\boldsymbol{\nabla}\left(\frac{1}{2} B^{2} \rho_{\alpha} \partial \tilde{\chi} / \partial \rho_{\alpha}\right)-\frac{1}{2} B^{2} \nabla \tilde{\chi} .
$$

This time, assuming $\tilde{\chi}$ as proportional to $\rho$, we obtain

$$
\mathbf{f}=M_{i} \boldsymbol{\nabla} B_{i},
$$

a result obviously different from Eq (1) - but one that also vanishes for uniform $B$-fields, so there is no disagreement to $\mathbf{f}=M_{i} \boldsymbol{\nabla} H_{i}$ in linear order.
Now, since Eqs (2) and (3) are algebraically equivalent, the difference must lie between the two seemingly innocuous assumptions, $\chi$ or $\tilde{\chi} \sim \rho$. Reviewing the above derivations, it is obvious that if one of the two assumptions were strictly correct, the other would be wrong, and only the associated force expression is applicable.

Generically, on the other hand, both $\chi$ and $\tilde{\chi}$ are power series of $\rho$. So we are simply approximating, discarding quadratic and higher order terms, when we assume that either is linear in $\rho$. The consistent dilute limit is given when all terms $\sim \rho^{2}$ (and higher) are discarded. With $\chi \sim \rho$, this necessarily implies that we must also discard all terms $\sim \chi^{2}$. As a result, $\tilde{\chi}=\chi /(1+\chi) \approx \chi$ and $M_{i} \boldsymbol{\nabla} B_{i} \approx M_{i} \boldsymbol{\nabla} H_{i}$. We conclude: The Kelvin force is valid to linear order in the density $\rho$ and the susceptibility $\chi$ (or magnetization $M_{i}$ ). Especially, both $M_{i} \boldsymbol{\nabla} B_{i}$ and $M_{i} \boldsymbol{\nabla} H_{i}$ are valid expressions for the Kelvin force.

The force in the experiment of [1] is of course finite. A proper, quantitative evaluation is given by including terms of higher order in $\rho$. As a first-step, we consider the next order terms:

$$
\begin{array}{r}
\chi=\alpha \rho(1+\beta \alpha \rho+\cdots), \\
\tilde{\chi}=\alpha \rho[1+(\beta-1) \alpha \rho+\cdots] .
\end{array}
$$

(Note that with $\chi, \tilde{\chi}=\alpha \rho$ in the dilute limit, $\alpha \rho$ is simply the sum of single particle contributions, from non-interacting dipoles.) Inserting these expansions into Eq (2) or (3), we find, for a constant $B$-field,

$$
\mathbf{f}=\frac{1}{2} B^{2} \nabla\left[(\beta-1)(\alpha \rho)^{2}\right] .
$$

There are four different microscopic models usually employed to calculate the temperature and concentration dependence of the susceptibility of ferrofluids: Weiss, Onsager, mean-spherical, and high-temperature. Though different in details, all provide the same value $\beta=1 / 3$, in good agreement with experimental data [3].

Assuming $\chi \sim \rho$ or $\tilde{\chi} \sim \rho$ to hold strictly is respectively equivalent to $\beta=0$ and $\beta=1$, with additional restrictions for the yet higher order terms. Both assumptions are arbitrary, and in stark contrast to our microscopic understanding of magnetism.

Mario Liu, Institut für Theoretische Physik, Universität Hannover, 30167 Hannover, Germany

[1] W. Luo, T. Du and J. Huang, Phys. Rev. Lett. 82, 4134 (1999)

[2] L.D. Landau and E.M. Lifshitz, Electrodynamics of Continuous Media (Pergamon, Oxford, 1984), §15

[3] A.F. Pshenishnikov, J. Magn. Magn. Mater. 145, 319 (1995) 